\title{
Novel Approach of New Dualistic Enhancement for Digital Image Segmentation using FCM
}

\author{
Amandeep Kaur \\ Research Scholar \\ Sachdeva Engg. College for \\ Girls, Gharuan (Mohali)
}

\author{
Rakesh Kumar \\ Dept. of CSE \\ Sachdeva Engg. College for \\ Girls, Gharuan (Mohali)
}

\author{
Sukjot Kaur \\ Dept. of CSE \\ Sachdeva Engg. College for \\ Girls, Gharuan (Mohali)
}

\begin{abstract}
Image segmentation plays important role in the biomedical images. The process of image segmentation is defined as the technique via which a given photograph is segmented into several parts in order to further analyze every of these components present in the photo [9]. In segmentation, without a doubt image is represented into greater understandable form. Segmentation essentially used to hit upon the gadgets, obstacles and other applicable facts in the digital snap shots. There are exceptional tactics to enforce segmentation like threshold, clustering and remodel strategies etc. [10]. The reason for the popularity of image segmentation is because of its importance in the area of image processing. The prime task of the researchers working in this field is to develop a method for efficient and better image segmentation. There are certain factors that affect the process of image segmentation like the intensity of image to be segmented, color, type and the noise present in the image [12]. No algorithm has been developed till date that could keep a look at all the above listed factors and then segment the image effectively so that all the problems that can come in the way of image segmentation can be avoided. The algorithm development for effective image segmentation is still a big research that will take place in the area of image processing. In this paper a new technique is proposed. This technique is capable of covering the lacking points of traditional techniques or algorithms. In this work DSIHE is implemented along with fuzzy C-Mean segmentation technique in order to enhance the quality and performance of the technique.
\end{abstract}

\section{Keywords}

Segmentation; Enhancement; Normalization Euclidean Distance; Signal to Noise Ratio; C-Mean Segmentation.

\section{INTRODUCTION}

In language of computers image segmentation is the process of dividing an image into small segments. These small segments are collection of pixels and also known as super pixels. The image segmentation is done in order to get more meaningful or simplified image. A simplified image is easy to understand and analyze. Image segmentation is a process in which the portion of the image which shares the same features are assigned with labels. The application of image segmentation in an image is to highlight or locate objects and boundaries like lines, curves etc. At last the obtained image includes the set of segments of contours which covers the whole image. Adjacent regions are significantly different with respect to the same properties. After applying segmentation the final image can be used to produce 3D effects by using interpolation algorithms. Medical imaging is a technique to generate such an image of human body which is helpful to doctors and scientists for the analysis. With the advancement in the technology many techniques are developed for the purpose of image segmentation but there is no appropriate solution exists for the problems of image segmentation. In order to generate a solution for problems of image segmentation these algorithms and techniques are concatenated with the domain knowledge. Image segmentation is a technique or process to divide an image into multiple segments due to which the explanation of segments can be transformed into a proper structure for the purpose of analysis and processing. There are various parameters like intensity of pixels, color, quality which helps to divide the picture into multiple segments. These segments identical to each other on the basis of above parameters. Some segmentation algorithms consider the property of discontinuity with sub region like edges, equality with the sub regions whereas some rely on both of the parameters. The image segmentation is different from the sample classification as the purpose of segmentation is to divide the image into various segments and the image classification is used to identify the divided segments. This is the only thing which differentiates both image segmentation and sample classification. Hence the process of image segmentation and sample classification are individual but sequential process. On the basis of image study problems and functioning of image segmentation, Sample classification and image processing can be used collectively. However the result of sample classification gets affected by image segmentation. And thus it leads to the instant analysis of the failure or success of the analysis process. Medical imaging, remote sensing, security surveillance, military object detection are some area in which sample classification and image analysis along with the segmentation is involved. The level of segmentation depends upon the segmentation problem to which extent it is solved. When the specific area (i.e. Region of Interest (ROI)) of the image is differentiated from the whole image then the process of image segmentation is stopped. In case of image study, independent segmentation is difficult to perform because of trouble depended property. The segmentation becomes more complicated when the image includes poor resolution due to noise and mixed pixels. Several techniques are available for image segmentation but the most suitable and appropriate technique is Active Contour. This method is used to validate the problems of segmentation and based on connectivitypreserving relaxation. This method is used for segmentation and object location in the image. It initially starts with the first boundary shape and then applies alterations on it by using contour evaluation (shrinkl expansion operation). Contour evaluation is used either by reducing the energy functions like fixed region based segmentation or by simulating the geometric fractional differential equations. The feature of dynamic contour is that it conquers an image into segments with continuous boundaries and border detectors are based on 
threshold and local filtering. Example of such is canny, continuous output in case of inconsistent boundaries. In case of active contours flexibility and convenience can be achieved by applying the level set theories. Various properties like edge, statistics and texture can be used by active contour according to variation in implementation method.

Image segmentation is important element in many sign processing method and its applications. The segmentation technique is to discover the higher positions of the shape factors consistent with the arrival records. The motive of image segmentation is to cluster pixels of a photo into photograph areas [20].

\section{APPLICATIONS OF IMAGE SEGMENTATION}

Image segmentation is applied in various fields. Some of the application fields are as follows:

2.1 Content-based image retrieval

2.2 Machine vision

2.3 Image segmentation in medical science

2.3.1 Tumor detection

2.3.2 Diagnosis etc.

2.4 Object locating and detection

2.4.1 Pedestrian detection

2.4.2 Face detection in various fields

2.4.3 Brake light detection

2.4.4 Object detection form satellite images as crops.

2.5 Biometric science

2.5.1 Face recognition system

2.5.2 Fingerprint identification system

2.6 Traffic control systems

\section{TECHNIQUES}

Many algorithms have been developed which can be used for the purpose of image segmentation. These techniques are based on domain specific knowledge in order to solve the domain segmentation issues. The techniques are categorized as follows:

\subsection{Thresholding}

It is the simplest method for image segmentation. The working of this technique is based on a threshold value defined by user. This technique is used to change an image from gray scale to binary image. In this technique a threshold value is used which is pre-defined. The methods which are based on this technique are entropy methods, k-mean clustering technique etc.

\subsection{Clustering based method}

This technique follows the idea of clustering. It divides the image into number of small k-clusters. The steps of this algorithm or technique are as follows:

1. Selection of k-cluster center either randomly or via some heuristic method.

2. In order to reduce the distance between pixels each pixel is allotted to the clusters or cluster centers.

3. After allocation of clusters, on the basis of average value cluster center is re-selected again.

4. Above 2 steps will repeat until the convergence is achieved.

In this method the distance refers to the difference between cluster centers and pixels. The difference is calculated on the basis of pixel intensity, color value of the pixel. The output or accuracy of the output relies on the value of $\mathrm{k}$ and set of clusters.

\subsection{Compression based method}

As the name suggest this technique is based on data compression. This is referred as the optimal technique for image segmentation. The reason behind its optimal nature is that it reduces all the variable segmentation and length of coded data. The logic behind this technique is that the segmentation is done by locating some matching patterns in the image and any regular data is used for compression. In this technique the segmentation is recognized by its consistency and outline that it follows. For available image segmentation, this technique bears that the large number of bits are needed to encode that the image is segmentation based. Then from various segmentation of the image the best segmentation is selected on the basis of reduced coding length.

\subsection{Histogram based}

This method is most preferable as compare to other. This technique is different from other techniques because in other technique at least one pass is required throughout the pixels of the image but in this technique a histogram is used for computation corresponding to all pixels. The peak and value of histogram helps to locate the clusters in the image.

An enhanced version of this technique is implemented on the clusters of the image for categorized them into various small clusters.

\subsection{Split and Merge technique}

The basic idea behind this technique is quad tree division of the image. Therefore another name is quad tree segmentation. The working of this technique is as follows:

The process starts from the root here root refers to the whole image. If the root is heterogeneous then it further divides it into four son-squares and so on. If the divided son-squares are homogeneous then they are merged as various linked sources. The nodes of the tree are segmented nodes. This procedure continues until no separation or merges are required.

List of some other methods is as follows:

1. Region Growing Method

2. Edge detection

3. Partial differential equation based technique

4. Parametric methods

5. Graph Partitioning

\section{METHODOLOGY}

In this section the methodology and block diagram of proposed technique is presented. In our proposed technique fuzzy C-Mean is applied for image enhancement. The methodology of proposed technique is as follows:

1. First step is to select the image from database.

2. Now DSIHE (Dualistic Sub Image Histogram Equalization) is applied for equalization. 
3. After equalization, Fuzzy C-Mean is applied for segmentation.

4. In this step the segmented image is generated.

5. After segmentation the result parameters are calculated.

6. After calculating performance parameters the comparison is performed for the purpose of efficiency measurement.

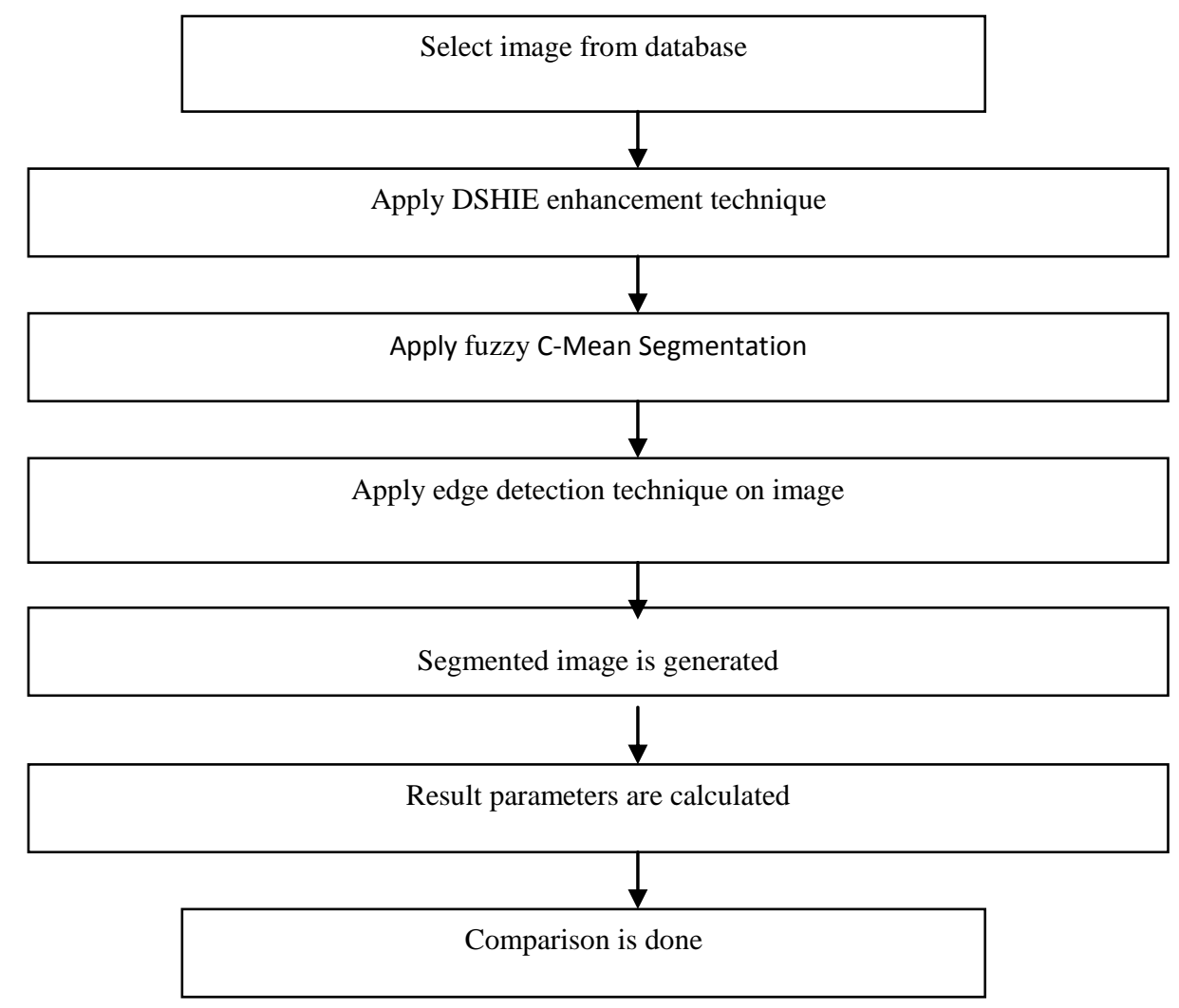

Figure 1. Block diagram of proposed technique

\section{PROBLEM FORMULATION}

Image segmentation is the process of segmenting the digital image into number of meaningful parts. In the conventional techniques like FCM the edges were not detected. Image enhancement was not done in the conventional techniques due to which the edges of the black \& white or colored images were not detected properly. Applying the image enhancement would preserve the edges of the images segmented and hence better images after segmentation will be obtained. The maximum entropy is used in spatial information for gray-level image threshold selection which yields generally unsatisfactory results. A new technique for image segmentation needs to be proposed that will apply image enhancement and then images will be segmented for obtaining satisfactory results.

\section{PROPOSED SYSTEM}

When an image is segmented into several parts and the parts ought to be meaningful, it is called as image segmentation. The conventional techniques had several drawbacks like edge preservation. A new technique is proposed in which image.

Enhancement will be done for preserving and detecting the edges. Using the technique of image enhancement the edges in the technique will be detected better than using the contour model. In proposed work as enhancement will be applied the sections of the image get cleared and will help in providing better segmentation are in as using the fuzzy approach in segmentation along with the C-mean clustering so can say that the approach that is proposed having the concept of image enhancement and the fuzzy along with the $\mathrm{c}$ mean clustering.

Applying on both techniques a better segmented image will be formed. This proposed technique for image segmentation yields satisfactory results and hence it is an efficient technique for image segmentation.

\section{Objectives}

1. To update the traditional segmentation approach with edge preserving

2. To introduce the concept of enhancement for better segmentation

3. To evaluate the performance of the system over segmentation.

\section{RESULTS AND DISCUSSIONS}

In this section the result of proposed technique is defines in order to prove the efficiency of the work. The efficiency of the technique is evaluated on the basis of various parameters 
such as SNR, NED, Average Error etc. The simulation is done by using MATLAB. Three images are used for implementing the proposed method.
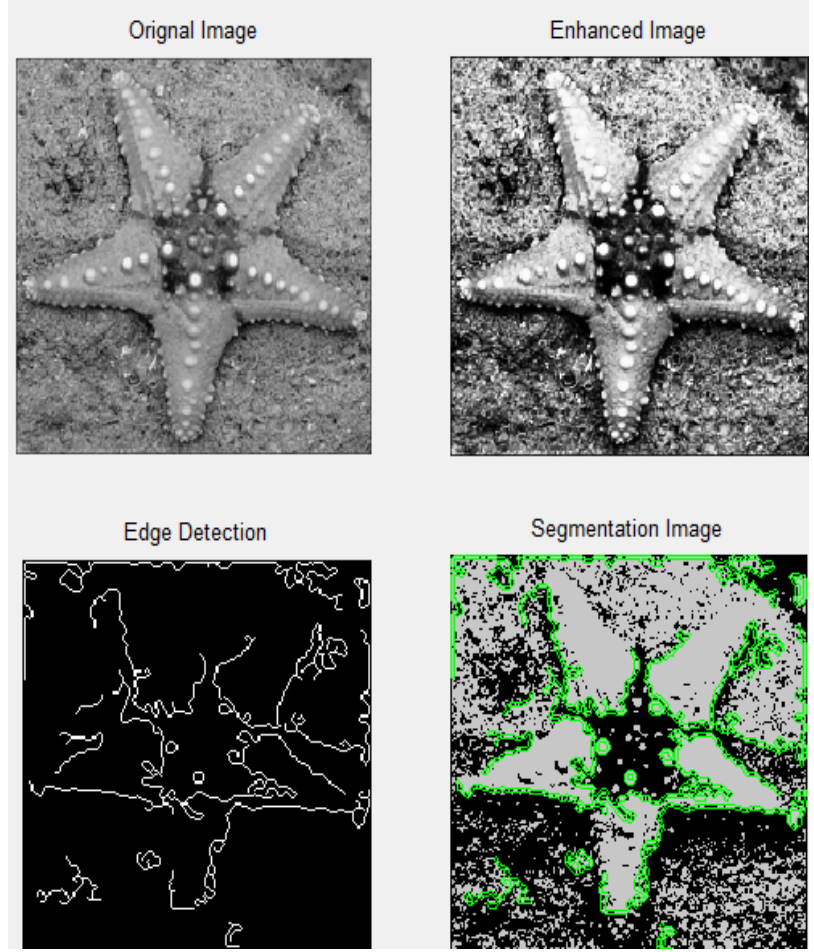

Figure 2. Image shows the image used for segmentation

In figure 2(original image) the image is represented which is selected for image segmentation. In above figure(original image) first image represents the original image, second (enhanced image) image represents the Enhanced image which is observed after applying DSHIE enhancement technique, third(edge detection) image is observed after applying edge detection, fourth (segmented image) image is the image which is observed after segmentation.

DSHIE stands for Dualistic Sub Image Histogram Equalization. This is a technique which divides the image into two parts on the basis of grey level PDF (Probability Density Function). Then the sub-parts of the image is processed which leads to the final image which is more enhanced as compare to the original image.

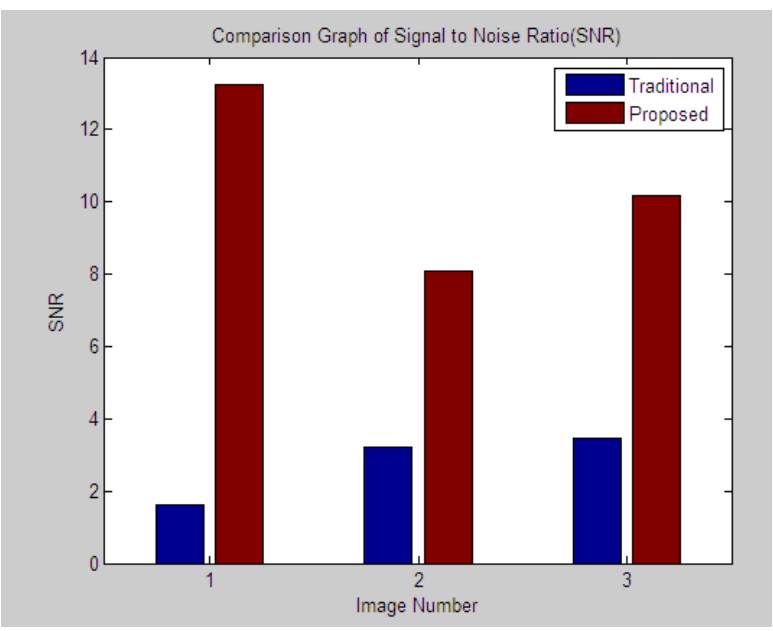

Figure 3.The SNR of 3 different images and compared with traditional technique
In figure 3 Graph the represents the comparison between traditional and proposed technique. This comparison is based on SNR i.e. Signal to Noise Ratio of the technique. The techniques are applied on various three images. It is observed that the SNR value of proposed technique in all images is quiet high as compare to the value of SNR in traditional technique.

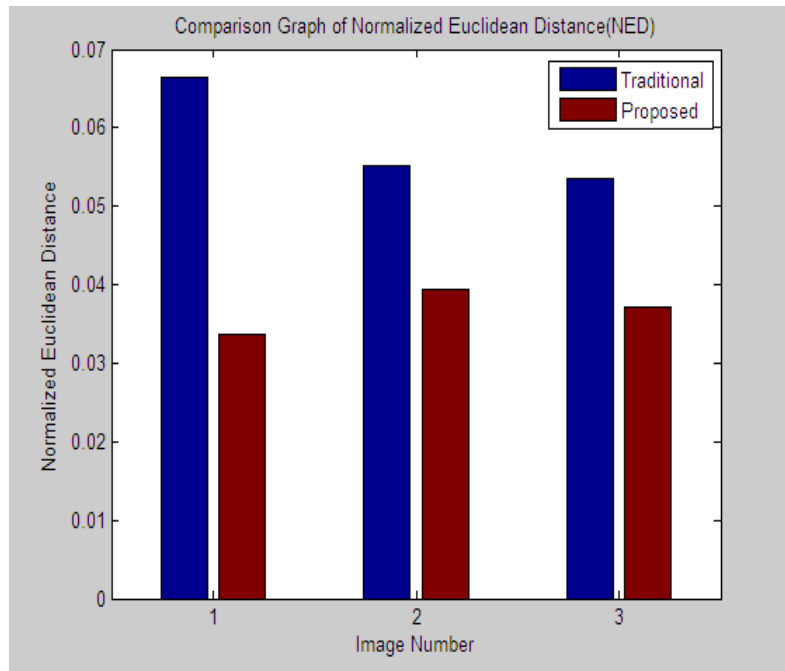

Figure 4.The NED of proposed and traditional technique

In figure 4 , Graph shows the comparison of proposed and traditional technique. The above graph shows the values of Normalization Euclidean Distance (NED). From above graph it is shown that the NED of proposed technique is less in all three images as compare to the proposed technique.

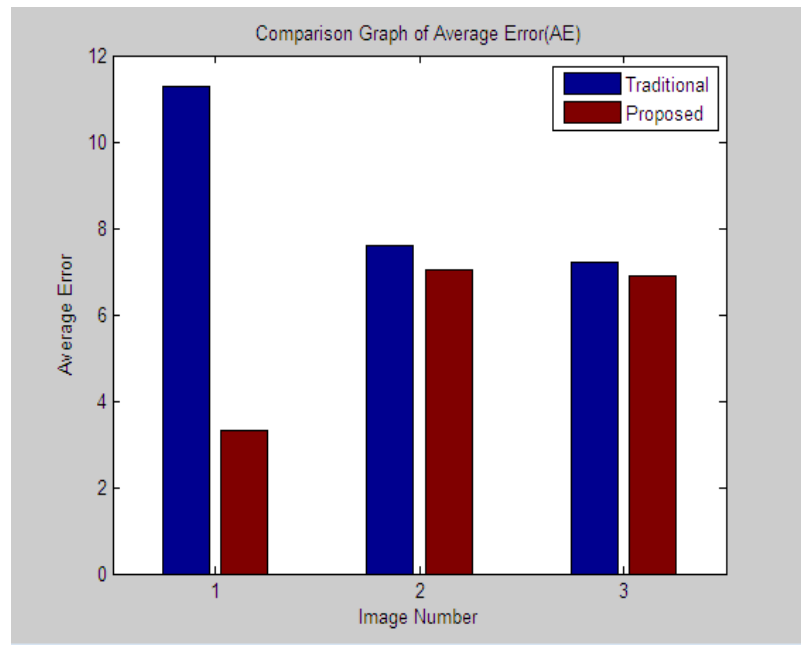

Figure 5 Graph compares the Average Error of proposed technique and traditional technique.

In figure 5 Graph shows the average error of proposed and traditional technique. This image shows that the average of generated error in particular technique. Number of average error in the technique should be low so that it can be ensure that the technique is quite efficient. It is observed that the average error value of proposed technique is low whereas in case of traditional technique this value is quite high. 


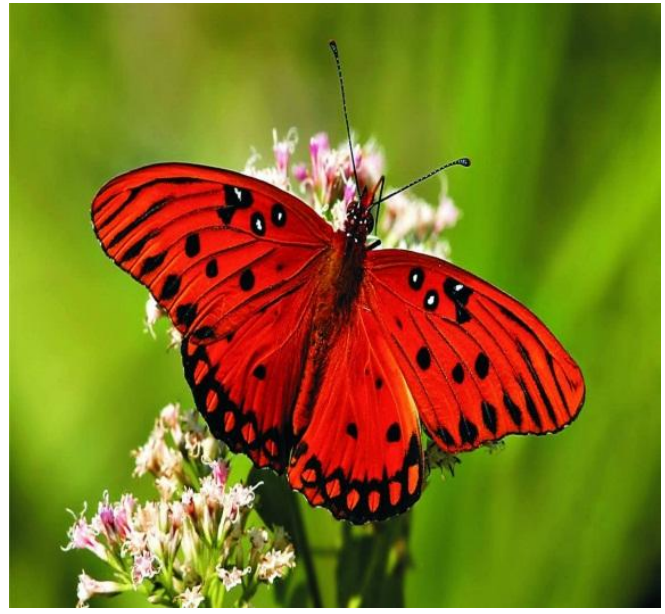

(A)

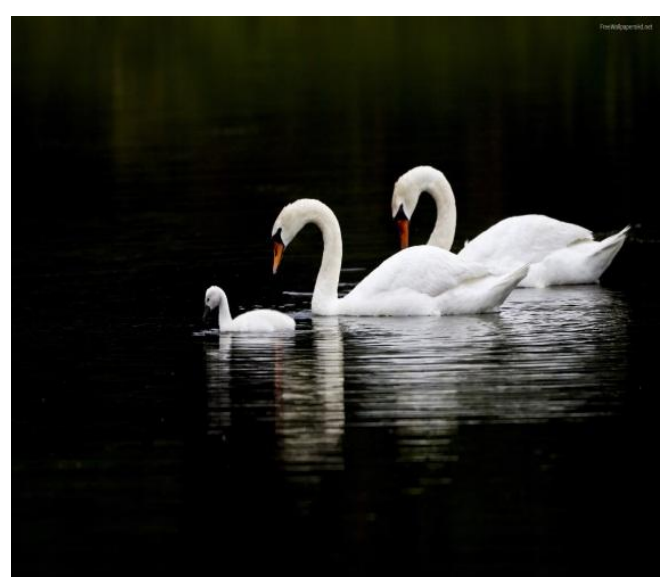

(B)

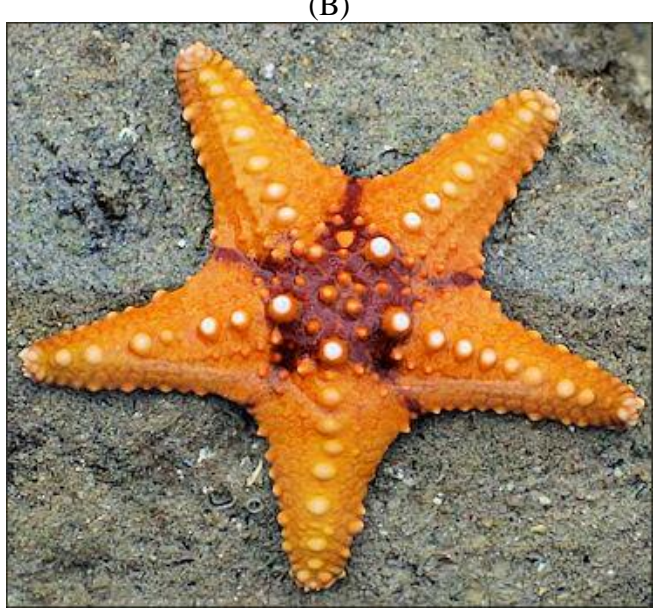

(C)

Figure 6.These images $(A, B, C)$ used for image segmentation

\section{CONCLUSION AND FUTURE SCOPE}

It is clearly shown that Signal to Noise ratio have higher value as well as Normalized Euclidean Distance and Average Error have lower values for all the sample images as compared to traditional approaches used for segmentation so proposed method is best and efficient. As a future scope further enhancement can be done in this method by using any trending technique of image segmentation. Along with this other image fusion technique can also be used that will fuse the image in efficient way so that the quality and information of the image is not degraded.

\section{REFERENCES}

[1] Alzate, C (April, 2007).Image Segmentation using a Weighted Kernel PCA Approach to Spectral Clustering.IEEE, CIISP 2007, pp 208-2013.

[2] Amanpreet Kaur(December 2014).A Review Paper on Image Segmentation and its Various Techniques in Image Processing.IJSR, Volume 3 Issue 12, pp 12-14.

[3] Ashraf A. Aly.research review for digital image segmentation techniques.ijcsit Vol 3, No 5, Oct 2011, pp 99-106.

[4] C.Sriramakrishnan (May, 2012).Performance Analysis of Advanced Image Segmentation Techniques.International Journal of Computer Applications, Volume 45- No.7, pp 13-18.

[5] Dibya Jyoti Bora (September, 2014).A Novel Approach Towards Clustering Based Image Segmentation.IJESE, Volume-2 Issue-11, pp 6-10.

[6] H C Sateesh Kumar,(February 2009).Automatic Image Segmentation using Wavelets.

[7] Hakeem Aejaz Aslam (March, 2013).A New Approach to Image Segmentation for Brain Tumor detection using Pillar K-means Algorithm.IJARCCE, Vol. 2, Issue 3, pp 1429-1436.

[8] Hui Zhang.Image Segmentation Evaluation.A Survey of Unsupervised Methods, Image.

[9] JITENDRA MALIK.Contour and Texture Analysis for Image Segmentation.International Journal of Computer Vision 43(1), 7-27, 2001.

[10] Khang Siang Tan (January, 2011) .Color image segmentation using histogram thresholding - Fuzzy Cmeans hybrid approach.ELSEVIER, VOl:44, Issue:1, pp $1-15$.

[11] M. Erd(2014).Segmentation: A New View of Image Segmentation and Registration.IEEE.

[12] M. Jogendra Kumar(September 2014).review on image segmentation techniques.ijsret, Volume 3, Issue 6 , pp 992-997.

[13] Mei Yeen Choong .An Image Segmentation using Normalised Cuts in Multistage Approach.IJSSST, pp 1016.

[14] Muhammad Waseem Khan et al(April 2014).A Survey: Image Segmentation Techniques. International Journal of Future Computer and Communication, Vol. 3, No. 2 , pp 89-93.

[15] N. Senthilkumaran( May 2009).Edge Detection Techniques for Image Segmentation - A Survey of Soft Computing Approaches. International Journal of Recent Trends in Engineering, Vol. 1, No. 2, pp 250-254.

[16] Pushpa .R. Suri, Mahak (June 2012).Image Segmentation With Modified K-Means Clustering Method.

[17] Rafika Harrabi (May, 2012).Color image segmentation using multi-level thresholding approach and data fusion techniques: application in the breast cancer cells images.Springer, pp 1-12. 
[18] Rajiv Kumar (December, 2011).Image Segmentation using Discontinuity-Based Approach.IJMIP, Volume 1, pp 72-78.

[19] Rohan Kandwal(April 2014).Review: Existing Image Segmentation Techniques.IJARCSSE, Volume 4, Issue 4 , pp 153-156.

[20] S Sapna Varshney(December 2009).Comparative study of image segmentation techniques and object matching using segmentation. IEEE, Methods and Models in Computer Sc2ience, 2009. ICM2CS 2009. Proceeding of International Conference on, pp 1-6.

[21] S.Dhanalakshmi(September 2012).A New Method for Image Segmentation.IJARCSSE, Volume 2, Issue 9, pp
293-299.

[22] Sharon Alpert.Image Segmentation by Probabilistic Bottom-Up Aggregation and Cue Integration.

[23] Shuai Zheng.Dense Semantic Image Segmentation with Objects and Attributes.

[24] V. Dey.A review on image segmentation techniques with remote sensing perspective.iaprs, Vol. XXXVIII, Part 7A, pp 31-42.

[25] Varshali Jaiswal (December, 2013).A Survey of Image Segmentation based on Artificial Intelligence and Evolutionary Approach.IOSR-JCE, Volume 15, Issue 3, PP 71-78. 